\title{
Salivary Level of Trace Element in Oral Lichen Planus, A Premalignant Condition
}

\author{
Fahimeh Rezazadeh $^{1 *}$, Sadaf Salehi ${ }^{2}$, Mostafa Rezaee ${ }^{1}$
}

\begin{abstract}
Background: Oral Lichen Planus (OLP) is a common inflammatory disease of unknown cause that knows also as a premalignant condition. Recent studies contributed nutritional factors to the pathogenesis of many autoimmune disorders. Objective: The aim of present study was to investigate the salivary levels of Magnesium ( $\mathrm{Mg}), \mathrm{Calcium}(\mathrm{Ca})$, Iron $(\mathrm{Fe})$, Zinc $(\mathrm{Zn})$ and Copper $(\mathrm{Cu})$ in this disorder. Materials and Methods: In this observational case-control study, the unstimulated saliva of 40 patients with OLP and 40 age and sex matched healthy control subjects were collected. The salivary levels of $\mathrm{Mg}, \mathrm{Ca}, \mathrm{Fe}, \mathrm{Zn}$ and $\mathrm{Cu}$ were determined using ICP-AES. Results: Mg levels were significantly lower in OLP patients than in healthy controls, although it didn't differ significantly between erosive and non-erosive types of Lichen Planus. Also there was no meaningful relationship between the levels of $\mathrm{Ca}, \mathrm{Fe}, \mathrm{Zn}$ and $\mathrm{Cu}$ in case and control groups. Conclusion: Trace elements such as Mg may have a role in ethiopathogenesis of OLP.
\end{abstract}

Keywords: Oral lichen planus- saliva- magnesium- calcium- iron- zinc- copper

Asian Pac J Cancer Prev, 20 (7), 2009-2013

\section{Introduction}

Lichen Planus is a common chronic inflammatory disease of the stratified squamous epithelium (Nima and FarzanehAgha, 2010). It mostly appears in oral mucosa (OLP) but involvement of other areas such as the skin, genitalia, nails, hair follicles, esophagus, urinary tract, nasal mucosa, larynx and even the eyes may be encountered in approximately $15-20 \%$ of the cases (Sugerman et al., 2002; Ayinampudi and Narsimhan, 2012).

There is a lack of valid epidemiologic studies regarding the prevalence of OLP but the overall prevalence as reported in the review by (Mc-Carthan and Healy, 2008) ranges from $0.47 \%$ to $1.27 \%$ with markedly women's predominance (Lavanya et al., 2011). Onset of the disease is usually at the 5th and 6th decades of life (Carbone et al., 2009; Rezazadeh et al., 2013), although younger adults and children might be affected (Alam and Hamburger, 2001). OLP has been introduced as a premalignant lesion, potentially leading to development of oral squamous cell carcinoma (OSCC) (Muñoz et al., 2007). Rate of the malignant transformation, as described in the literature, varies between $0.04 \%$ and $1.74 \%$ per year (Van der Meij et al., 2003). The most prevalent form of OLP is the reticular form, which is diagnosed by the presence of white hyperkeratotic lines and dots known as Wickham's striae (Thorn et al., 1988). Other types include papular, bullous, and ulcerative (Neville et al., 2015).

The etiology of OLP is not entirely known, but it is believed that autoreactive T cells play the most important role in pathogenesis of the disease (Burket et al., 2008; Rezazadeh et al., 2017). Findings suggest that basal keratinocyte antigens associated with MHC class I may activate lesional cytotoxic CD8 $+\mathrm{T}$ cells, consequently promoting keratinocyte apoptosis (Farhi and Dupin, 2010). Several risk factors such as drugs, systemic disorders, trauma, viral infections like Hepatitis $\mathrm{C}$ and stress have also been suggested in association with the pathogenesis of the disease (Sugerman et al., 2002).

Nutrition and nutritional status have been found to have a major effect on immune functions, resistance to infection and autoimmunity (Harbige, 1996). Trace elements such as Magnesium (Mg), Calcium (Ca), Iron (Fe), Zinc ( $\mathrm{Zn})$ and Copper $(\mathrm{Cu})$ although present in minute quantities are required for maintenance of life, growth, reproduction, and prevention of diseases (Sandström, 2001). Magnesium exerts a large variety of biological functions, including structural roles, controlling roles, and regulatory roles (Tam et al., 2003). Some studies have illustrated that low levels of $\mathrm{Mg}$ may be associated with hyperactivity of macrophages and neutrophils (Tam et al., 2003). Also it has been reported that $\mathrm{Mg}$ insufficiency may contribute to OSMF and SCC development (Al-Rawi and Talabani, 2005; Kode and Karjodkar, 2013; Hosthor et al., 2014). Calcium plays a very important role in cell signaling, which is the basis of all cellular functions. Errors in signaling interactions and cellular information processing can contribute to cancer, autoimmune diseases 
and diabetes (Solinas et al., 2007; Wang et al., 2012; Vlahopoulos et al., 2015). Dysregulation in Ca responses have been reported in association with pathophysiology of several autoimmune and inflammatory diseases (Rezazadeh et al., 2017). Iron is essential in antioxidant processes, in a way that alteration in iron concentration may cause formation of reactive oxygen species (ROS), which the negative roles have been proven in different autoimmune diseases (Sepici-Dinçel et al., 2006; Prasad, 2009). Reduced amount of iron has also been reported in cancer patients (Stevens et al., 1988). Zinc is associated with protein and nucleic acid production, and regulating $\mathrm{T}$ lymphocyte's function (Lukaski, 2004). Some studies have also reported zinc insufficiency in autoimmune diseases such as diabetes and pemphigus vulgaris (Kazi et al., 2008; Yazdanpanah et al., 2011). Copper acts as an important cofactor for some metalloenzymes, which are necessary for oxidation-reduction activities (Taysi et al., 2003). Alteration of $\mathrm{Cu}$ concentration has been reported effective in different disorders such as leukoplakia, pemphigus vulgaris, OSMF and SCC (Al-Rawi and Talabani, 2005; Yazdanpanah et al., 2011; Hosthor et al., 2014; Kumar et al., 2014; Shetty et al., 2015). According to our knowledge, reports on the role of trace elements in OLP patients are few and conflicting (Challacombe, 1986; Kleier et al., 1998; Ayinampudi and Narsimhan, 2012; Gholizadeh et al., 2014; Chen et al., 2015; Rezazadeh and Abbasi, 2015) and no similar study has evaluated salivary level of these trace elements in OLP patients. Therefore, we conducted a study to measure the salivary concentration of $\mathrm{Mg}, \mathrm{Ca}$, $\mathrm{Fe}, \mathrm{Zn}$ and $\mathrm{Cu}$ in order to find any relationship between these micronutrients and the disorder.

\section{Materials and Methods}

This case-control study was performed on oral lichen planus patients who were referred to the oral medicine department of Shiraz Dental School (Shiraz, Iran) between August 2016 and March 2017. The diagnosis was based on clinical examination, in which Wickham's striae was seen. However, a biopsy was conducted in patients with atypical lesions. None of our patients, had skin lesion. Patients with signs of other lichenoid reactions such as contact lichenoid reaction and drug induced lichenoid reaction were excluded from the study. Also the exclusion criteria applied to patients who had other autoimmune diseases, concurrent inflammation, malignancies or presence of epithelial dysplasia in pathology report, and patients who were taking systemic drugs, vitamins or mineral supplements in the past 2 months. Eventually, forty eligible patients with OLP were enrolled to study. Statistician determine minimum 34 patients and most of previous article also had lower sample size $(10,5,20, \ldots)$ so we select 40 sample size. Forty age and sex-matched healthy subjects who were referred to Shiraz Dental School for routine dental procedure were included as control group. Patients also were divided into two groups of erosive oral lichen planus and non-erosive oral lichen planus.

Saliva sampling was conducted in the morning and subjects were instructed not to consume any food or drink 2 hours before sample collection (Rezazadeh et al., 2017). Participants were asked to rinse their mouth using water, and subsequently $5-10 \mathrm{ml}$ non- stimulated whole saliva was collected from the oral cavity, where it was allowed to accumulate at the floor of the mouth, and then transferred to a sterile glass tube. The samples were frozen at -20 until the time of analysis. $10 \mathrm{ml} \mathrm{HNO} 3$ and $15 \mathrm{ml}$ deionized water were added to the samples and burned at $170 \mathrm{PSI}$ and $200^{\circ} \mathrm{C}$ in a microwave. In this way, the specimen was dissolved. After calibration of the vehicle, $2 \mathrm{ml}$ specimens were taken.

The levels of five elements-magnesium, calcium, iron, zinc and copper- in each specimen were analyzed using the ICP-AES technique (715ES, Varian, Palo Alto, USA). The samples were aspirated in a nebulizer and carried by a stream of argon directed centrally toward the hole in the vehicle. The samples were heated by conduction and radiation and reached $10,000^{\circ} \mathrm{C}$, where they were completely atomized. At this point they emit light of a characteristic wavelength. Thus, every element was described according to its different wave- lengths (Table 1). The mineral content of each sample was measured as mg/Lit (PPM).

The study protocol was accepted by Ethics Committee of Shiraz University of Medical Sciences, and each subject signed a written informed consent form. SPSS software (version 20.0; SPSS Inc, Chicago, IL, USA) was used for statistical analyses. Results were analyzed according to Mann-Whitney U test and Kruskal-Wallis. A p-value $<0.05$ was regarded as statistically significant.

\section{Results}

A total of 80 subjects were recruited to the study: 40 patients diagnosed with oral lichen planus and 40 age and sex matched individuals. The patient group consisted of 26 females and 14 males with the mean age of $46.6 \pm 11.85$, and the control group included 31 females and 9 males with $46.06 \pm 12.13$ mean of age. The median and mean salivary concentrations of Magnesium $(\mathrm{Mg})$, Calcium $(\mathrm{Ca})$, Iron $(\mathrm{Fe})$, Zinc $(\mathrm{Zn})$ and Copper $(\mathrm{Cu})$ are presented in Table 2. Mean magnesium level was significantly lower in patients than healthy control subjects $(\mathrm{p}=0.000)$. However, the differences were not significant for other elements $(\mathrm{p}>0.05)$.

The average salivary level of studied elements, showed no significant difference between erosive OLP patients (18 patients) and non-erosive OLP patients (22 patients) (Table 3). Only Mg level in each group (erosive and non-erosive) showed a significant difference with control group $(\mathrm{p}=0.000)$.

\section{Discussion}

Oral lichen planus is an inflammatory muco-cutaneous

Table 1. Different Elements wave Lengths

\begin{tabular}{lccccc}
\hline Element & $\mathrm{Mg}$ & $\mathrm{Zn}$ & $\mathrm{Ca}$ & $\mathrm{Fe}$ & $\mathrm{Cu}$ \\
\hline $\begin{array}{l}\text { Wave length } \\
(\mathrm{nm})\end{array}$ & 279.553 & 213.857 & 370.602 & 238.204 & 324.754 \\
\hline
\end{tabular}


Table 2. The Salivary Levels of Trace Elements in Healthy and Patient Groups

\begin{tabular}{lccc}
\hline $\begin{array}{l}\text { Variable } \\
(\mathrm{mmol} / \mathrm{L}) \#\end{array}$ & Healthy group & OLP group & P value* \\
\hline $\mathrm{Cu}$ & 0.3 & 0.02 & 0.704 \\
& $(0.30 \pm 0.01)$ & $(0.02 \pm 0.01)$ & \\
$\mathrm{Fe}$ & 0.09 & 0.07 & 0.557 \\
& $(0.11 \pm 0.10)$ & $(0.16 \pm 0.24)$ & \\
$\mathrm{Zn}$ & 1.03 & 1.11 & 0.981 \\
& $(1.58 \pm 1.60)$ & $(1.60 \pm 1.53)$ & \\
$\mathrm{Ca}$ & 19.51 & 19.58 & 0.736 \\
& $(28.39 \pm 28.77)$ & $(21.80 \pm 14.93)$ & \\
$\mathrm{Mg}$ & 199.88 & 84.41 & 0 \\
& $(332.76 \pm 394.38)$ & $(105.40 \pm 77.16)$ & \\
\hline
\end{tabular}

Median (Mean \pm SD); *Mann-Whitney U test; \# mmol/L, milimol per liter

disorder. Despite all the research efforts, etiologic factors are yet to be fully known (Mehdipour et al., 2015). Recently studies have drawn attention to the possible involvement of trace elements alterations in pathogenesis and progress of the disease (Maggini et al., 2007; Chen et al., 2015; Mehdipour et al., 2015). Furthermore, saliva is one of the body fluids, which is collected easily and non-invasively. Therefore, Several studies have recently addressed the potential properties of saliva in diagnosing oral and systemic diseases (Teles et al., 2009). We investigated salivary level of magnesium, calcium, iron, zinc and copper in patients suffering from OLP in order to study the possible relationship between these elements and the disease.

Our study seems to be the first to assess salivary Magnesium (Mg) content in OLP patients. The results showed a significant decrease in salivary levels of $\mathrm{Mg}$ in patients compared to healthy subjects. However, no significant difference was observed between $\mathrm{Mg}$ amount of erosive and non-erosive lichen planus patients. Magnesium is one of the most abundant cations in cellular systems, which is responsible for a large variety of biological functions. This element also plays a key role in the immune system; that is, as a co-factor for immunoglobulin synthesis, C'3 convertase, antibody-dependent cytolysis, immune cell adherence, IgM lymphocyte binding, macrophage response to lymphokines and $\mathrm{T}$ helper-B cell adherence. Animal studies have observed an increase in the level of inflammatory cytokines such as IL-6 and TNF-, due to $\mathrm{Mg}$ insufficiency. Also higher levels of macrophage and neutrophil activities have been reported as a result of magnesium deprivation (Kleier et al., 1998; Tam et al., 2003). So based on these vital roles of $\mathrm{Mg}$ in immune system especially $\mathrm{T}$ cells and our results, reduction in $\mathrm{Mg}$ level may be an etiologic factor in development of OLP. This result is in accordance with Kode and Karjodkar, (2013) and Al-Rawi et al., (2005) studies that evaluated salivary $\mathrm{Mg}$ levels in oral submucous fibrosis (OSMF) and squamous cell carcinoma (SCC) patients, and reported a decrease in the amount of salivary $\mathrm{Mg}$ in patients compared to controls. Other studies assessed serum levels of $\mathrm{Mg}$ in different disorders. Hosthor et al.,
Table 3. The Level of Salivary Trace Elements in the Crosive and Non-Erosive Groups

\begin{tabular}{lcc}
\hline Variable $(\mathrm{mmol} / \mathrm{L}) \#$ & Non-erosive & Erosive \\
\hline $\mathrm{Cu}$ & 0.02 & 0.02 \\
& $(0.02 \pm 0.01)$ & $(0.03 \pm 0.01)$ \\
$\mathrm{Fe}$ & 0.05 & 0.09 \\
& $(0.13 \pm 0.23)$ & $(0.19 \pm 0.25)$ \\
$\mathrm{Ca}$ & 15.95 & 21.22 \\
& $(19.80 \pm 12.23)$ & $(24.25 \pm 17.75)$ \\
$\mathrm{Zn}$ & 1.05 & 1.25 \\
& $(1.36 \pm 1.59)$ & $(1.90 \pm 1.43)$ \\
$\mathrm{Mg}$ & 76.14 & 101.6 \\
& $(92.71 \pm 57.82)$ & $(120.92 \pm 95.20)$ \\
\hline
\end{tabular}

Median (Mean \pm SD); \# mmol/L, milimol per liter

(2014) evaluated serum concentration of $\mathrm{Mg}$ in OSMF and SCC patients. Level of Mg had decreased significantly in both groups of patients. Yilmaz et al., (2005) also reported a decrease in serum $\mathrm{Mg}$ concentration in lupus erythematous (SLE) patients. In contrast, Shpitzer et al., (2007) reported an increase in the amount of Mg in saliva of 25 SCC cases. This conflict may be related to lower number of participants and the different disorders that were evaluated.

Present study showed lower salivary Zinc (Zn) levels in patients with OLP than controls but the difference was not significant which is collinear with the result of Rezazadeh and Abbasi, (2015) and kleier et al., (1998) that reported no significant difference between serum amounts of $\mathrm{Zn}$ in OLP patients and control group. Even though another study by Ayinampudi and Narsimhan, (2012) reported a significant increase in salivary $\mathrm{Zn}$ in patients with pre-malignancies, including 5 OLP patients This can be explained by the low number of participants in this study. Gholizadeh et al., (2014) also concluded that serum Zn decreases in OLP subjects, this difference may be related to usage of serum in this research rather than saliva. Serum and saliva concentration of $\mathrm{Zn}$ has also been evaluated in disorders like leukoplakia, OSMF and SCC. The result of these studies showed a decreased $\mathrm{Zn}$ level in patients compared to controls (Yilmaz et al., 2005; Yazdanpanah et al., 2011; Hosthor et al., 2014; Kumar et al., 2014; Shetty et al., 2014; Shetty et al., 2015). This conflict can be due to the difference in the studied disorders.

Based on our results, mean level of Copper $(\mathrm{Cu})$ was approximately equal between the two groups, and no significant difference was observed, which is similar to the result of Kode and Karjodkar, (2013) study on OSMF patients. However, study of Ayinampudi and Narsimhan, (2012) on OLP patients reported a significant increase in this elements salivary concentration, which can be again related to the low number of subjects ( 5 cases) studied in this research. Other researches have also been conducted in order to evaluate the $\mathrm{Cu}$ level in serum and saliva of leukoplakia, SLE, OSMF and SCC patients. Shetty et al., (2015), Yilmaz et al., (2005), Baharvand et al., (2014), and Hosthor et al., (2014) reported significant increase in the amount of this element but Al-Rawi et al., (2005) Kumar et al., (2014) and Yazdanpanah et al., (2011) reported Asian Pacific Journal of Cancer Prevention, Vol 202011 
opposing results (Hosthor et al., 2014; Kumar et al., 2014; Shetty et al., 2015). This can be explained by the fact that they studied disorders other than OLP.

This study showed reduced amounts of Iron (Fe) in the case group but the results were not significantly different from the control group. In Kode and Karjodkar, (2013). study as well, no significant difference was seen between salivary Fe level of OSMF patients and controls. Kumar et al., (2014) studies also reported that there is no relationship between serum iron and leukoplakia and SCC. However, Shetty et al., (2015) and Al-Rawi and Talabani, (2005) reported a reduction in the salivary levels of Fe in SCC and OSMF patients compared to healthy subjects. Difference in the studied cases and used techniques may be responsible for this conflict. Some studies have also measured Fe level in serum of patients suffering from SCC, Baharvand et al., (2014) observed an elevation in this elements serum level; however a decrease was reported in some cases of SLE, OSMF and SCC (Yilmaz et al., 2005, Kode and Karjodkar, 2013; Hosthor et al., 2014).

Calcium (Ca) levels in this study were elevated in OLP patients; however, this elevation was not significant. Our study seems to be the first to evaluate salivary $\mathrm{Ca}$ levels in OLP patients. Hosthor et al conducted a study on the serum level of this element in OSMF patients and didn't observe a significant difference; although, a meaningful increase was seen in SCC patients (Hosthor et al., 2014). Shpitzer et al., (2007) also reported an elevation in salivary Ca level of SCC patients. This can be explained by use of serum, different techniques and also the difference in disorders being studied. However, in line with our result, Nola-Fuchs et al., (2011) reported no relationship between salivary Ca level and SCC.

Results of this study indicated that Mg salivary levels were significantly lower in OLP patients compared to healthy subjects; however, there was no relationship between this element and the type of OLP. No correlation was observed regarding other elements and the disorder. Therefore, it seems that $\mathrm{Mg}$ may be effective in etiology of OLP. Overall, different results also may be related to nutrient status of patients that none of these studies adverted to it. So we suggest that future studies evaluate nutritional status and also assess serum and salivary level concurrently in larger sample sizes. Comparison of trace elements between OLP lesions with dysplasia and non-dysplastic OLP lesions also is warranted.

\section{Acknowledgments}

The authors thank the vice-chancellery of Shiraz University of Medical Science for supporting this research. This article is based on the thesis by Sadaf Salehi from Shiraz Dental School (Grant\#11562). The authors also thank Dr Vosough of the Dental Research Development Center for statistical analysis.

\section{References}

Al-Rawi NH, Talabani N (2005). Quantitative analysis of trace elements in saliva of oral cancer patients from Iraq. J College
Dentistry, 17, 32-5.

Alam F, Hamburger J (2001). Oral mucosal lichen planus in children. Int J Paediatr Dent, 11, 209-14.

Ayinampudi BK, Narsimhan M (2012). Salivary copper and zinc levels in oral pre-malignant and malignant lesions. J Oral Maxill Pathol, 16, 178.

Baharvand M, Manifar S, Akkafan R, Mortazavi H, Sabour S (2014). Serum levels of ferritin, copper, and zinc in patients with oral cancer. Biomed J, 37, 331-6.

Burket LW (2015). Burket's oral medicine, red and white lesions, PMPH-USA. Philadelphia, 5, 57-68.

Carbone M, Arduino P, Carrozzo M, et al (2009). Course of oral lichen planus: a retrospective study of 808 northern Italian patients. Oral Dis, 15, 235-43.

Challacombe S (1986). Haematological abnormalities in oral lichen planus, candidiasis, leukoplakia and nonspecific stomatitis. Int J Oral Maxill Surg, 15, 72-80.

Chen H-M, Wang Y-P, Chang JY-F, et al (2015). Significant association of deficiencies of hemoglobin, iron, folic acid, and vitamin $\mathrm{B}_{12}$ and high homocysteine level with oral lichen planus. J Form Med Assoc, 114, 124-9.

Fahimeh R, Neshat A, Jannan G (2013). Oral lichen planus resistency to topical corticosteroid therapy. Middle-East $J$ Scien Res, 16, 1245-8.

Farhi D, Dupin N (2010). Pathophysiology, etiologic factors, and clinical management of oral lichen planus, part I: facts and controversies. Clin Dermatol, 28, 100-8.

Gholizadeh N, Mehdipour M, Najafi S, et al (2014). Evaluation of the serum zinc level in erosive and non-erosive oral lichen planus. J Dent, 15, 52.

Harbige LS (1996). Nutrition and immunity with emphasis on infection and autoimmune disease. Nutr Health, 10, 285-312.

Hosthor SS, Mahesh P, Priya SA (2014). Quantitative analysis of serum levels of trace elements in patients with oral submucous fibrosis and oral squamous cell carcinoma: A randomized cross-sectional study. J Oral Maxill Pathol, 18, 46.

Kazi TG, Afridi HI, Kazi N, et al (2008). Copper, chromium, manganese, iron, nickel, and zinc levels in biological samples of diabetes mellitus patients. Bio Trace Elem Res, 122, 1-18.

Kleier C, Werkmeister R, Joos U (1998). Zinc and vitamin A deficiency in diseases of the mouth mucosa. Mund Kiefer Gesich, 2, 320-5.

Kode MA, Karjodkar FR (2013). Estimation of the serum and the salivary trace elements in OSMF patients. J Clin Diag Res, 7, 1215.

Kumar A, Poojary D, Darji H, KS R (2014). Estimation of serum micronutrient levels and the possible risk of oral cancer and premalignancy. Int J Innov Res Scie Engin Techn, 3, 8360-3.

Lavanya N, Jayanthi P, Rao UK, Ranganathan K (2011). Oral lichen planus: An update on pathogenesis and treatment. J Oral Maxill Pathol, 15, 127.

Lukaski HC (2004). Vitamin and mineral status: effects on physical performance. J Nutr, 20, 632-44.

Maggini S, Wintergerst ES, Beveridge S, Hornig DH (2007). Selected vitamins and trace elements support immune function by strengthening epithelial barriers and cellular and humoral immune responses. Br J Nutr, 98, 29-35.

McCartan B, Healy C (2008). The reported prevalence of oral lichen planus: a review and critique. J Oral Pathol Med, 37, 447-53.

Mehdipour M, Zonoz AT, Kohnavard M, Gholizadeh N, Namazi $Z$ (2015). Evaluation of the serum calcium level in erosive oral lichen planus. Sci J Hamadan Univ Med Sci, 22, 65-70.

Muñoz AA, Haddad RI, Woo S-B, Bhattacharyya N (2007). 
Behavior of oral squamous cell carcinoma in subjects with prior lichen planus. Otolaryngol Head Neck Surg, 136, 401-4.

Neville BW (2015). Oral and maxillofacial pathology, dermathologic disease, Elsevier Health Sciences, St. Louis. Missouri, 63043, 654-9.

Nima M, FarzanehAgha H (2010). Hepatitis C virus and lichen planus: the real association. Hep Monthly, 2010, 161-4.

Nola Fuchs P, Rogić D, Vidović-Juras D, et al (2011). Salivary analytes in patients with oral squamous cell carcinoma. Colleg Antropol, 35, 359-62.

Prasad AS (2009). Impact of the discovery of human zinc deficiency on health. J Am Coll Nutr, 28, 257-65.

Rezazadeh F, Abbasi E (2015). Evaluation of serum Zinc level in oral lichen planus, a case control study. Elixir Dent, 84, 33627-9.

Rezazadeh F, Shahbazi F, Andisheh-Tadbir A (2017). Evaluation of salivary level of IL-10 in patients with oral lichen planus, a preliminary investigation. Comp Clin Pathol, 26, 531-4.

Sandström B (2001). Micronutrient interactions: effects on absorption and bioavailability. Br J Nutr, 85, 181-5.

Sepici-Dinçel A, Özkan Y, Yardim-Akaydin S, et al (2006). The association between total antioxidant status and oxidative stress in Behçet's disease. Rheumatol Int, 26, 1005.

Shetty SR, Babu SG, Rao PK, et al (2014). Interdependence of antioxidants and micronutrients in oral cancer and potentially malignant oral disorders: a serum and saliva study. J Dent (Tehran), 11, 696-702.

Shpitzer T, Bahar G, Feinmesser R, Nagler RM (2007). A comprehensive salivary analysis for oral cancer diagnosis. $J$ Cancer Res Clin Oncol, 133, 613-7.

Solinas G, Vilcu C, Neels JG, et al (2007). JNK1 in hematopoietically derived cells contributes to diet-induced inflammation and insulin resistance without affecting obesity. Cell Metab, 6, 386-97.

Stevens RG, Jones DY, Micozzi MS, Taylor PR (1988). Body iron stores and the risk of cancer. NEng JMed, 319, 1047-52.

Sugerman PB, Savage NW, Walsh LJ, et al (2002). The pathogenesis of oral lichen planus. Crit Rev Oral Biol Med, 13, 350-5.

Tam M, Gómez S, González-Gross M, Marcos A (2003). Possible roles of magnesium on the immune system. Eur J Clin Nutr, 57, 1193-7.

Taysi S, Gulcin I, Sari RA, Kuskay S, Bakan N (2003). Trace elements and disease activity score in patients with rheumatoid arthritis. Pain Clin, 15, 435-9.

Teles RP, Likhari V, Socransky SS, Haffajee AD (2009). Salivary cytokine levels in subjects with chronic periodontitis and in periodontally healthy individuals: a cross-sectional study. J Periodontal Res, 44, 411-7.

Thorn JJ, Holmstrup P, Rindum J, Pindborg JJ (1988). Course of various clinical forms of oral lichen planus. A prospective follow-up study of 611 patients. J Oral Pathol Med, 17, 213-8.

van der Meij EH, Schepman KP, van der Waal I (2003). The possible premalignant character of oral lichen planus and oral lichenoid lesions: a prospective study. Oral Surg Oral Med Oral Pathol Oral Radiol Endodontol, 96, 164-71.

Vlahopoulos SA, Cen O, Hengen N, et al (2015). Dynamic aberrant NF- $\mathrm{B}$ spurs tumorigenesis: A new model encompassing the microenvironment. Cytokine Growth Factor Rev, 26, 389-403.

Wang K, Grivennikov SI, Karin M (2012). Implications of anti-cytokine therapy in colorectal cancer and autoimmune diseases. Ann Rheum Dis, 2012, 202201.

Yazdanpanah MJ, Ghayour-Mobarhan M, Taji A, et al (2011). Serum zinc and copper status in Iranian patients with pemphigus vulgaris. Int J Dermatol, 50, 1343-6.

Yilmaz A, Sari RA, Gundogdu M, Kose N, Dag E (2005). Trace elements and some extracellular antioxidant proteins levels in serum of patients with systemiclupus erythematosus. Clin Rheumatol, 24, 331-5.

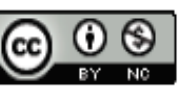

This work is licensed under a Creative Commons AttributionNon Commercial 4.0 International License. 